\title{
Quantum critical phenomena of long-range interacting bosons in a time-dependent random potential
}

\author{
Kihong Kim凶 \\ Department of Physics, Ajou University, Suwon 442-749, Korea
}

\begin{abstract}
We study the superfluid-insulator transition of a particle-hole symmetric system of long-range interacting bosons in a time-dependent random potential in two dimensions, using the momentum-shell renormalization-group method. We find a new stable fixed point with non-zero values of the parameters representing the short- and long-range interactions and disorder when the interaction is asymptotically logarithmic. This is contrasted to the non-random case with a logarithmic interaction, where the transition is argued to be firstorder, and to the $1 / r$ Coulomb interaction case, where either a first-order transition or an XY-like transition is possible depending on the parameters. We propose that our model may be relevant in studying the vortex liquidvortex glass transition of interacting vortex lines in point-disordered type-II superconductors.
\end{abstract}

PACS Numbers: 05.30.Jp, 64.60.Ak, 74.40.+k, 74.60.Ge

Typeset using REVTEX 
In recent years, there has been great interest in quantum critical phenomena occurring

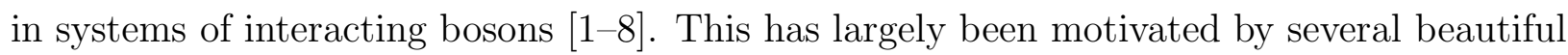
experiments on the superconductor to insulator transition in thin metallic films at very low temperature [9 14]. In many models, the superconductor (or charged superfluid) to insulator (or Bose glass) transition in disordered films is considered as a localization transition of bosonic Cooper pairs in an external random potential [1, 目团. In non-random systems, the superfluid to (Mott) insulator transition can be triggered by changing the density of bosons through critical values commensurate with the underlying periodic lattice [1.2]. Since Cooper pairs are charged, one needs to consider the influence of long-range interactions on the nature of the critical phenomena. In the absence of disorder, the superconductor-Mott insulator transition of charged bosons in two dimensions interacting by the $1 / r$ Coulomb potential has been studied by Fisher and Grinstein via renormalization-group methods [1]. They showed that when the (bare) Coulomb interaction is sufficiently weak, the transition belongs to the three-dimensional XY-model universality class, whereas when the Coulomb interaction is sufficiently strong, it is first-order. In this paper, we generalize Fisher and Grinstein's model to the cases with the $1 / r$ or logarithmic interaction in a time-dependent random potential. Time dependence of the potential in classical static critical phenomena is unimportant [8]. In quantum critical phenomena, however, it can lead to new universality classes as will be shown in the present work. As well as being relevant in studying novel quantum critical phenomena, our model may be also a useful representation of a system of long-range interacting vortex lines in point-disordered type-II superconductors in an external magnetic field. According to the mapping originally due to Nelson, vortex lines in three spatial dimensions are considered as the world lines of bosons residing in two spatial dimensions perpendicular to the magnetic field [15] [17]. The dimension parallel to the magnetic field is mapped to the imaginary time dimension of a bosonic system. Therefore the static random potential provided by isotropically distributed impurities in superconductors can be regarded as a time-dependent potential that is random in both space and time. The quantum superconductor-insulator transition of interacting bosons is analogous to the clas- 
sical vortex liquid-vortex glass transition of interacting vortex lines. We caution that in our model, the long-range interaction between vortex lines is confined to the plane perpendicular to the magnetic field. Applying the standard momentum-shell renormalization-group method to the logarithmically interacting case, we discover a new stable fixed point in two dimensions with non-zero values in the short- and long-range interactions and disorder when there exists a time-dependent random potential, whereas we find a first-order transition in the non-random case. Since the interaction between vortex lines is logarithmic in a wide range of length scales when the magnetic field is close to the upper critical field [18], we propose a possibility that our new random fixed point may describe the real vortex liquidvortex glass transition in point-disordered type-II superconductors. On the other hand, when the interaction is the $1 / r$ Coulomb interaction, we find a behavior analogous to Fisher and Grinstein's results in a non-random system.

We consider the $(d+1)$-dimensional classical action for the $m$-component classical complex Bose field $\psi_{i}(\vec{x}, \tau)$ coupled to the scalar gauge field $A(\vec{x}, \tau)$ mediating the long-range interaction between charge $e$ bosons in $d$ spatial and one temporal dimensions:

$$
\begin{aligned}
S= & \int d^{d} x \int d \tau \sum_{i=1}^{m}\left[a\left|\left(\partial_{\tau}-i e A\right) \psi_{i}\right|^{2}+b\left|\nabla \psi_{i}\right|^{2}\right. \\
& \left.+r(\vec{x}, \tau)\left|\psi_{i}\right|^{2}\right]+\int d^{d} x \int d \tau \sum_{i, j=1}^{m} u\left|\psi_{i}\right|^{2}\left|\psi_{j}\right|^{2} \\
+ & \frac{1}{2} \sum_{\vec{k}, \omega}\left(c k^{\sigma}+g k^{2}\right)|A(\vec{k}, \omega)|^{2}
\end{aligned}
$$

where $A(\vec{k}, \omega)$ is the Fourier transform of $A(\vec{x}, \tau) . a, b, u, c$ and $g$ are positive constants and $r(\vec{x}, \tau)$ is a Gaussian random function satisfying

$$
\begin{aligned}
& r(\vec{x}, \tau)=r_{0}+\delta r(\vec{x}, \tau), \quad\langle\delta r(\vec{x}, \tau)\rangle=0, \\
& \left\langle\delta r(\vec{x}, \tau) \delta r\left(\vec{x}^{\prime}, \tau^{\prime}\right)\right\rangle=2 v \delta\left(\vec{x}-\vec{x}^{\prime}\right) \delta\left(\tau-\tau^{\prime}\right),
\end{aligned}
$$

where $\langle\cdots\rangle$ denotes the statistical averaging and $v(>0)$ measures the strength of randomness. The action $S$ applies to the cases where there is a particle-hole symmetry and the interaction potential between bosons is proportional to $1 / r^{d-\sigma}$ in the long distance limit, $r$ 
being the distance between bosons. The $1 / r$ Coulomb interaction corresponds to $\sigma=d-1$, while the asymptotically logarithmic interaction corresponds to $\sigma=d$. In the absence of the particle-hole symmetry, one has to replace the term proportional to $a$ in (11) by a term of the form $\psi_{i}{ }^{*}\left(\partial_{\tau}-i e A\right) \psi_{i}$ [2.3]. In general, disordered systems do not possess the particle-hole symmetry. We note, however, that it has been argued recently, in a rather compelling manner, that the particle-hole asymmetric term is irrelevant in the renormalization group sense and the exact (statistical) particle-hole symmetry is restored after averaging over disorder at the critical points of various kinds of disorder-driven superfluid transitions, including the vortex glass transition [19 22]. We accept these arguments and assume that the particle-hole symmetric action, Eq. (11), describes the critical fixed point of the vortex glass transition. When $r$ is non-random and $g$ is zero, the superconductor to Mott insulator transition described by this model was studied by Fisher and Grinstein [1]. In the present paper, we generalize their work to the cases where $r$ is a Gaussian random function of both space and time.

We use the standard replica trick to reduce $S$ to a non-random effective action:

$$
\begin{aligned}
S_{\mathrm{eff}}= & \int d^{d} x \int d \tau \sum_{\alpha=1}^{p} \sum_{i=1}^{m}\left[a\left|\left(\partial_{\tau}-i e A_{\alpha}\right) \psi_{\alpha i}\right|^{2}\right. \\
& \left.+b\left|\nabla \psi_{\alpha i}\right|^{2}+r_{0}\left|\psi_{\alpha i}\right|^{2}\right] \\
+ & \int d^{d} x \int d \tau \sum_{\alpha=1}^{p} \sum_{i, j=1}^{m} u\left|\psi_{\alpha i}\right|^{2}\left|\psi_{\alpha j}\right|^{2} \\
& -\int d^{d} x \int d \tau \sum_{\alpha, \gamma=1}^{p} \sum_{i, j=1}^{m} v\left|\psi_{\alpha i}\right|^{2}\left|\psi_{\gamma j}\right|^{2} \\
+ & \frac{1}{2} \sum_{\alpha=1}^{p} \sum_{\vec{k}, \omega}\left(c k^{\sigma}+g k^{2}\right)\left|A_{\alpha}(\vec{k}, \omega)\right|^{2},
\end{aligned}
$$

where $\alpha$ and $\gamma$ are replica indices and the number of replica $p$ is sent to zero at the end of the calculation. In order to apply the momentum shell renormalization group method, we perform a Fourier transform of Eq. (3). The Fourier-transformed action has five types of interaction vertices. We perform a perturbation expansion in terms of these vertices to one loop order and integrate out the components with wave number $\vec{k}$ in a shell $k_{\Lambda} / e^{l}<k<k_{\Lambda}$, where $k_{\Lambda}$ is the momentum space cutoff and $e^{l}$ is the rescaling parameter. We recover the 
original form of the action by renormalizing $r_{0}, a, b, g, u$ and $v$ using the results of the momentum space integration and rescaling frequency, momentum and the fields with the rules

$$
\begin{aligned}
& \vec{k} \rightarrow \vec{k} / e^{l}, \quad \omega \rightarrow \omega / e^{z l} \\
& \psi_{\alpha i}(\vec{k}, \omega) \rightarrow \psi_{\alpha i}(\vec{k}, \omega) e^{\left(\zeta_{\psi}+\frac{d}{2}+\frac{z}{2}\right) l}, \\
& A_{\alpha}(\vec{k}, \omega) \rightarrow A_{\alpha}(\vec{k}, \omega) e^{\left(\zeta_{A}+\frac{d}{2}+\frac{z}{2}\right) l},
\end{aligned}
$$

where $z$ is the dynamical exponent and $\zeta_{\psi}$ and $\zeta_{A}$ are rescaling factors of the fields.

We perform our calculations in an $\epsilon(=3-d)$ expansion about the upper critical dimension $d=3$. Firstly, we consider the competition of the $k^{\sigma}$ and $k^{2}$ terms in the gauge field propagator. A naive power counting suggests that the $k^{2}$ term is irrelevant as long as the $k^{\sigma}$ term with $0<\sigma<2$ is present. This conclusion is not always true, however, since the $k^{2}$ term is renormalized in a perturbation theory, whereas the nonanalytic $k^{\sigma}$ term is not, and in some cases, this renormalization makes the $k^{2}$ term more relevant than the $k^{\sigma}$ term. To confirm this, we fix $a=b=g=1$ and perform a perturbation calculation assuming that $c$ is irrelevant. To one loop order, we obtain the exponents

$$
z=1-\frac{\tilde{w}}{3}, \quad 2 \zeta_{\psi}=1-d+\frac{\tilde{w}}{6}
$$

and the recursion relations for $c$ :

$$
\frac{d c}{d l}=\left(2-\sigma-\frac{m}{12} \tilde{w}\right) c
$$

and for $r_{0}, u$ and $v$ :

$$
\begin{aligned}
& \frac{d r_{0}}{d l}=\left(2-\frac{\tilde{w}}{6}\right) r_{0}+\frac{4(m+1) \tilde{u}}{\sqrt{1+r_{0}}}-\frac{4 \tilde{v}}{\sqrt{1+r_{0}}} \\
&+\frac{\tilde{w} \sqrt{1+r_{0}}}{2}, \\
& \frac{d \tilde{u}}{d l}= \epsilon \tilde{u}-2(m+4) \tilde{u}^{2}+12 \tilde{u} \tilde{v}+\frac{\tilde{u} \tilde{w}}{2}-\frac{\tilde{w}^{2}}{16}, \\
& \frac{d \tilde{v}}{d l}=\epsilon \tilde{v}+8 \tilde{v}^{2}-4(m+1) \tilde{u} \tilde{v}+\frac{\tilde{v} \tilde{w}}{2}
\end{aligned}
$$


where $\tilde{u}=u \kappa_{d} / 4, \tilde{v}=v \kappa_{d} / 4, \tilde{w}=w \kappa_{d}=e^{2} \kappa_{d}$ and $\kappa_{d}=2 /(4 \pi)^{d / 2} \Gamma(d / 2)$. The momentum cutoff $k_{\Lambda}$ was chosen to be equal to 1 . The recursion relation for the parameter $w \equiv e^{2}$ is obtained from the condition that the action is invariant under the gauge transformation

$$
\psi=\tilde{\psi} e^{i e \lambda}, \quad A=\tilde{A}+\frac{d \lambda}{d \tau}
$$

for any function $\lambda(\tau)$ to all orders. We find

$$
\frac{d \tilde{w}}{d l}=\left(2 \zeta_{A}+2 z\right) \tilde{w}=\epsilon \tilde{w}-\left(\frac{m+4}{12}\right) \tilde{w}^{2}
$$

which possesses a stable fixed point $\tilde{w}^{*}=12 \epsilon /(m+4)$. Substituting this value into Eq. (6), we observe that the parameter $c$ is indeed irrelevant at this fixed point if $2>\sigma>\sigma_{c}=$ $2-m \tilde{w}^{*} / 12=2-m \epsilon /(m+4)$. In the cases with $0<\sigma<\sigma_{c}$, where $g$ is irrelevant, we fix $a=b=c=1$ and $g=0$ and perform a one-loop calculation. It turns out that the recursion relations (7) remain the same, whereas Eq. (9) is replaced by

$$
\frac{d \tilde{w}}{d l}=\left(\epsilon-\epsilon_{\sigma}\right) \tilde{w}-\frac{\tilde{w}^{2}}{3},
$$

where we have introduced another small parameter $\epsilon_{\sigma}=2-\sigma$.

We consider the $1 / r$ Coulomb interaction case first. According to the results obtained above, the $k^{2}$ term in the gauge field propagator is irrelevant to the first order in $\epsilon$ in the physically interesting case with $\epsilon=1$ (that is, $d=2$ ) and $m=1$. Then $\epsilon-\epsilon_{\sigma}=1-d+\sigma=0$ and the marginally irrelevant parameter $\tilde{w}$ satisfies

$$
\frac{d \tilde{w}}{d l}=-\frac{\tilde{w}^{2}}{3}
$$

Integrating this equation and the recursion relations for $\tilde{u}$ and $\tilde{v}$ numerically for various initial values of $\tilde{u}, \tilde{v}$ and $\tilde{w}$, we find the renormalization group flow diagram qualitatively similar to that discovered by Fisher and Grinstein. That is, there is a plane in the parameter space separating the domain of attraction to the $(4-\epsilon)$-dimensional random XY-model fixed point with positive $\tilde{u}^{*}$ and $\tilde{v}^{*}$ and zero $\tilde{w}^{*}$ [23] and the domain where all points flow to the region with large negative $\tilde{u}$, large positive $\tilde{v}$ and zero $\tilde{w}$. When the physical value of $\tilde{w}$ is 
sufficiently small, the flows are attracted to the XY fixed point. When the initial $\tilde{w}$ value is large, $\tilde{u}$ grows to a large negative value, $\tilde{v}$ grows to a large positive value and the effective action (3) becomes thermodynamically unstable. This behavior is usually interpreted as a signature of a fluctuation-driven first-order phase transition. We illustrate this behavior by showing the flow diagram in the non-random case with $\tilde{v}=0$ in Fig. 1.

In case of the logarithmic interaction in two dimensions, we have only the $k^{2}$ term in the gauge field propagator. In this case, the effective charge parameter $\tilde{w}$ has a stable fixed point at $\tilde{w}^{*}=12 \epsilon /(m+4)$. Let us consider the non-random case with $\tilde{v}=0$ first. Then it is easy to show that $\tilde{u}$ possesses a stable fixed point at $\tilde{u}^{*}=\left(m+10+\sqrt{m^{2}-52 m-188}\right) \epsilon / 4(m+2)^{2}$ only when $m>m_{c}=26+12 \sqrt{6} \approx 55.394$. When $m$ is smaller than $m_{c}$, all points flow to large negative $\tilde{u}$ values as shown in Fig. 2. We cautiously interpret this as a signature of a first-order phase transition [24]. In the random case, we substitute the fixed point value of $\tilde{w}$ into the recursion relations for $\tilde{u}$ and $\tilde{v}$ and find a stable fixed point with positive $\tilde{u}^{*}, \tilde{v}^{*}$ and $\tilde{w}^{*}$ when $m=1$. The fixed point values are

$$
\tilde{u}^{*}=\frac{11+\sqrt{409}}{40} \epsilon, \quad \tilde{v}^{*}=\frac{\sqrt{409}}{40} \epsilon, \quad \tilde{w}^{*}=\frac{12}{5} \epsilon .
$$

This point has three irrelevant eigenvalues $-\epsilon$ and $-(33 \pm \sqrt{2183} i) \epsilon / 20$. The complex eigenvalues suggest that the renormalization-group flows spiral into the stable fixed point and give oscillatory corrections to scaling. In Fig. 3, we show the flow diagram in the $(\tilde{u}, \tilde{v})$ plane with $\tilde{w}$ fixed at $\tilde{w}^{*}$. There is a separatrix dividing the domain of attraction to the random fixed point and the unstable region. The critical exponents associated with the new random fixed point are

$$
\begin{aligned}
& \eta=\frac{2}{5} \epsilon, \quad z=1-\frac{4}{5} \epsilon, \quad \frac{1}{\nu}=2-\left(\frac{9}{10}+\frac{\sqrt{409}}{20}\right) \epsilon, \\
& \frac{1}{\nu_{\tau}}=\frac{1}{z \nu}=2+\left(\frac{7}{10}-\frac{\sqrt{409}}{20}\right) \epsilon,
\end{aligned}
$$

where $\nu$ and $\nu_{\tau}$ are the correlation length and time exponents and $\eta$ is defined by $2 \zeta_{\psi}+d+z=$ $2-\eta$ 
In conclusion, we performed a renormalization-group analysis of the superfluid-insulator transition in a particle-hole symmetric model of long-range interacting bosons with or without a time-dependent random potential. We find a new stable fixed point with non-zero disorder and long-range interaction when the interaction is logarithmic. Without a random potential, the transition in logarithmically interacting systems is argued to be first-order. We propose a possibility that our model may be relevant in interpreting the vortex liquid-vortex glass transition of interacting vortex lines in type-II superconductors.

This work has been supported by the Korea Science and Engineering Foundation through grant number 961-0209-052-2. 


\section{REFERENCES}

* Electronic address: khkim@madang.ajou.ac.kr

[1] M. P. A. Fisher and G. Grinstein, Phys. Rev. Lett. 60, 208 (1988).

[2] M. P. A. Fisher, P. B. Weichman, G. Grinstein, and D. S. Fisher, Phys. Rev. B 40, 546 (1989).

[3] P. B. Weichman and K. Kim, Phys. Rev. B 40, 813 (1989).

[4] M. P. A. Fisher, G. Grinstein, and S. M. Girvin, Phys. Rev. Lett. 64, 587 (1990).

[5] M. P. A. Fisher, Phys. Rev. Lett. 65, 923 (1990).

[6] K. Kim and P. B. Weichman, Phys. Rev. B 43, 13583 (1991).

[7] M.-C. Cha, M. P. A. Fisher, S. M. Girvin, M. Wallin, and A. P. Young, Phys. Rev. B 44, 6883 (1991).

[8] S. L. Sondhi, S. M. Girvin, J. P. Carini, and D. Shahar, Rev. Mod. Phys. 69, 315 (1997).

[9] D. B. Haviland, Y. Liu, and A. M. Goldman, Phys. Rev. Lett. 62, 2180 (1989).

[10] A. F. Hebard and M. A. Paalanen, Phys. Rev. Lett. 65, 927 (1990).

[11] Y. Liu, D. B. Haviland, B. Nease, and A. M. Goldman, Phys. Rev. B 47, 5931 (1993).

[12] A. Yazdani and A. Kapitulnik, Phys. Rev. Lett. 74, 3037 (1995).

[13] N. Marković, C. Christiansen, and A. M. Goldman, preprint cond-mat/9808132.

[14] N. Mason and A. Kapitulnik, preprint cond-mat/9810228.

[15] D. R. Nelson, Phys. Rev. Lett. 69, 1973 (1988).

[16] M. P. A. Fisher and D. H. Lee, Phys. Rev. B 39, 2756 (1989).

[17] R. A. Lehrer and D. R. Nelson, preprint cond-mat/9806016. 
[18] D. Huse and L. Radzihovsky, in Proceedings of 1993 Altenberg Summer School, Fundamental Problems in Statistical Mechanics VIII, edited by H. van Beijeren and M. H. Ernst (Elsevier, Amsterdam).

[19] M. P. A. Fisher, Physica A 177, 553 (1991).

[20] A. T. Dorsey and M. P. A. Fisher, Phys. Rev. Lett. 68, 694 (1992).

[21] R. Mukhopadhyay and P. B. Weichman, Phys. Rev. Lett. 76, 2977 (1996).

[22] F. Pázmándi and G. T. Zimányi, Phys. Rev. B 57, 5044 (1998).

[23] According to the Harris criterion [A. B. Harris, J. Phys. C 7, 1671 (1974)], in two spatial dimensions where $\epsilon=1$, this random fixed point is actually unstable with respect to the critical fixed point of the pure XY model, since the exact specific heat exponent $\alpha$ of the pure three-dimensional XY model takes a slightly negative value. Therefore the transition in two dimensions belongs to the pure XY-model universality class.

[24] The flow diagram in the clean logarithmic case resembles very much that of the classical zero-field superconducting transition in $(4-\epsilon)$ dimensions [B. I. Halperin, T. C. Lubensky, and S. K. Ma, Phys. Rev. Lett. 32, 292 (1972)], which, in fact, has been argued to be a second-order transition in three dimensions where $\epsilon=1$ and belong to the universality class of the inverted three-dimensional XY-model [C. Dasgupta and B. I. Halperin, Phys. Rev. Lett. 47, 1556 (1981)]. This suggests that the one-loop epsilon expansion in the present case may break down in two spatial dimensions. Even if it turns out that the superfluid-Mott insulator transition in the clean logarithmic case is second-order in two dimensions, we do not expect it is described by the three-dimensional XY-model fixed point, because the clean logarithmic model is self-dual in the long distance limit, whereas the XY model is not [5, []. 


\section{FIGURES}

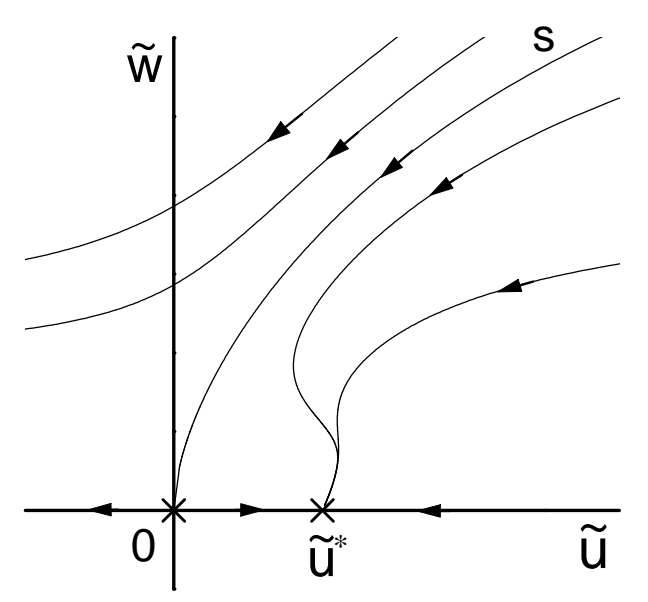

FIG. 1. Flow diagram for the non-random Bose system interacting by the $1 / r$ Coulomb potential when $m=1$. $S$ labels the separatrix dividing the domain of attraction to the XY fixed point and the unstable region. 


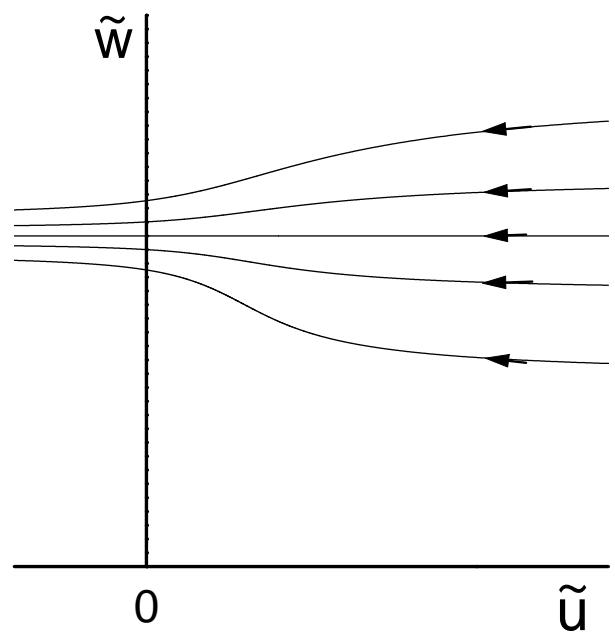

FIG. 2. Flow diagram for the logarithmically-interacting non-random Bose system when $m=1$. 


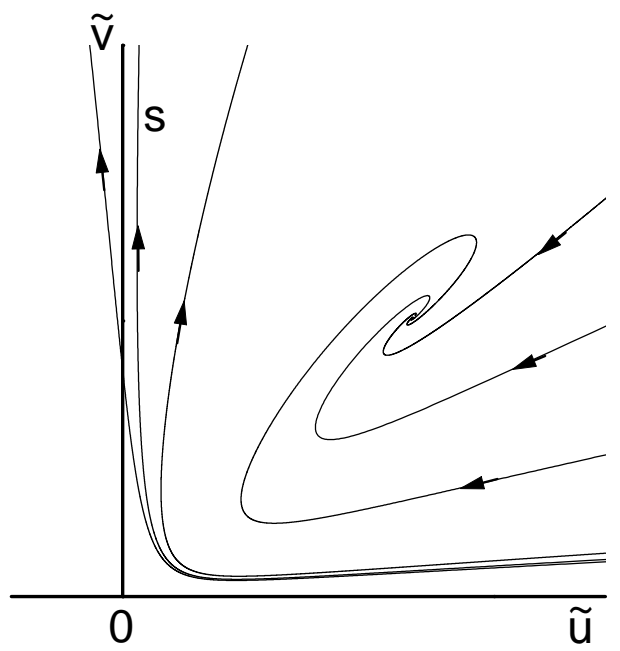

FIG. 3. Flow diagram for the logarithmically-interacting Bose system in a time-dependent random potential when $m=1$ and $\tilde{w}=\tilde{w}^{*}=12 \epsilon / 5$. S labels the separatrix dividing the domain of attraction to the random fixed point with positive $\tilde{u}, \tilde{v}$ and $\tilde{w}$ values and the unstable region. 